\section{A Simplified Method for Formulation of Epoxy Resin Embedding Media}

\author{
E. Ann Ellis \\ Texas A\&M University, College Station, TX \\ eann.ellis@worldnet.att.net
}

In a recent paper on the revised formulation of Spurr low viscosity embedding medium with ERL $4221^{1}$ the importance of maintaining an appropriate anhydride:epoxide (A:E) ratio was discussed. By understanding a few simple concepts about epoxy resin formulations and setting up a formulation table it is possible to create new resin mixtures with good sectioning properties and other desirable properties such as decreased viscosity and increased beam stability.

Before starting a formulation you need to know the molecular weight of the anhydride and the WPE (weight per epoxide equivalent $)^{2}$ of the epoxy resin component. The molecular weights and WPEs are usually printed on the bottle or can be obtained from the vendor. An A:E ratio of 0.7:1.0 -1.0:1.0 is used for most biological specimens. Increasing the A:E ratio results in a harder block; decreasing the A:E ratio results in a softer block. Table 1 shows a basic formulation spreadsheet where the molecular weights of the anhydrides and the WPEs of the epoxy resin components can be entered. The A:E ratio is entered under the anhydride for the molar ratio and the molar ratios of the epoxy components are entered under the epoxy components. The calculations are done as shown in each column and row.

TABLE 1: Formulation Table for Epoxy Resin Mixtures

\begin{tabular}{|c|c|c|c|}
\hline COMPONENT & DDSA & Araldite 502 & Quetol 651 \\
\hline Mol. Wt. or WPEA & 266 & $225^{\mathrm{A}}$ & $87 \mathrm{~A}$ \\
\hline $\begin{array}{l}\text { A:E Ratio }{ }^{\mathrm{B}} \text { or Molar } \\
\text { RatioC }^{\mathrm{C}}\end{array}$ & $1.0^{\mathrm{B}}$ & $0.5 \mathrm{C}$ & $0.5 \mathrm{C}$ \\
\hline Proportion by Wt. & $266 \times 1.0=266$ & $225 \times 0.5=112.5$ & $87 \times 0.5=43.5$ \\
\hline Total Wt. & $\begin{array}{c}266+112.5+ \\
43.5=422\end{array}$ & & \\
\hline$\%$ by Wt. & $\begin{array}{c}266 / 422= \\
63.0 \%\end{array}$ & $112.5 / 422=26.7 \%$ & $\begin{array}{c}43.5 / 422= \\
10.3 \%\end{array}$ \\
\hline $\begin{array}{l}\text { Formulation in } \\
\text { Grams }\end{array}$ & $6.3 \mathrm{~g}$ & $2.67 \mathrm{~g}$ & $1.03 \mathrm{~g}$ \\
\hline
\end{tabular}

The molar ratio is not the same as the A:E ratio. The molar ratio involves the relation of two components in the same class, either anhydrides or epoxides. For example, the total ratio of A:E is 1.0:1.0; since there are two epoxy components involved to give 1.0 for the epoxide component of the ratio, the molar ratios for Araldite and Quetol can be any combination that gives a total of 1.0 ( 0.4 for Araldite and 0.6 for Quetol or 0.5 and 0.5 for each). When using two anhydrides, the same principle applies as explained for the epoxide components. The formulation method is based on percentage by weight so that any amount of resin mixture can be made by multiplying the percentage by weight for each component by the total weight of resin mixture.

Formulations of epoxy resin mixtures were necessary when Luft's Epon $^{3}$ was introduced since the WPE of Epon 812 varied greatly from batch to batch. Now that the WPE's of most epoxides are fairly consistent, it is not necessary to calculate new formulations that often. I use the formulation method to vary the viscosity or hardness of a mixture for a particular type of specimen. In the formulation example given above, I used Quetol 651, a low viscosity, straight chain epoxy resin, to lower the viscosity of an Epon-Araldite mixture and maintain the good sectioning properties of a firm to hard block. ${ }^{4}$ The table below gives the viscosities of the standard formulations and those of new formulations with Quetol 651 to reduce the viscosity of the working formulation. I normally use benzyldimethylamine (BDMA) as the accelerator for all epoxy resin mixtures at the rate of $0.20 \mathrm{ml} / 10$ grams of resin mixture.

TABLE 2: Viscosity of Different Resin Formulations

\begin{tabular}{|l|c|}
\hline RESIN FORMULATION & VISCOSITY IN CENTIPOISE \\
\hline EPON 812-ARALDITE 502 & 2500 \\
\hline QUETOL 651-ARALDITE 502 & 800 \\
\hline LUFT'S EPON 812 & 550 \\
\hline LX-112 LUFT'S FORMULA & 340 \\
\hline QUETOL 651-LX-112 & 250 \\
\hline ERL 4221 SPURR & 180 \\
\hline QUETOL 651-ERL 4221 SPURR & 100 \\
\hline VCD SPURR & 60 \\
\hline
\end{tabular}

The formulations in Table 2 were made on the basis of an A: E ratio of 1.0:1.0 and a molar ratio for each epoxides of 0.5 for the Araldite containing formulations. The Luft's Epon ${ }^{3}$ formulation used an A:E ratio of 0.7:1.0 with the molar ratio of nadic methyl anhydride equal to 0.35 and the molar ratio of dodecenyl succinic anhydride equal to 0.35 . The combined molar ratios of the two anhydrides equal $0.7[0.35+0.35=0.70]$. The Spurr resin ${ }^{5}$ based formulations used an A:E ratio of $0.88: 1.0$ as given in the original Spurr publication.

The significance of maintaining an appropriate A:E ratio for a given embedding formulation ${ }^{2}$ has not been appreciated in recent years. The formulation originally proposed by Luft using dodecenyl succinic anhydride, nadic methyl anhydride and Epon 812 had an A: E ratio of 0.7:1.0; the Spurr low viscosity formulation had a ratio of 0.88:1.0. Experience over the past thirty years in my lab has shown that an A:E ratio of 0.9-1.0:1.0 gives a good quality block when using Epon-Araldite combinations. In cases where I use an Epon 812 substitute in Luft's formulation, I have found that raising the A:E ratio to $0.9: 1.0$ generally results in a block with better sectioning quality.

Audrey Glauert has reported in her publications over the years and I have observed likewise that the benzene ring structure of Araldite based formulations and the cyclic structures of ERL 4206 and ERL 4221 give better beam stability than those of the straight chain Epon substitutes. I often use formulation methods and combinations of cyclic and/or benzene ring based epoxides with the straight chain Quetol 651 to produce resin mixtures with lower viscosity and better beam stability. Generally, I use only one anhydride, either DDSA or NSA, for a given mixture since anhydrides absorb water more readily than the other resin components. A single source of anhydride makes it easier to identify a wet component if such should happen. As in the case of all resin mixtures, I prepare a small batch of resin and polymerize it to check the overall properties before I commit to infiltrating and embedding specimens.

\section{References}

1. E. A. Ellis, Microscopy Today 14 32(2006)

2. A. M. Glauert and P. R. Lewis, Chapt. 6 Embedding in epoxy resins in Biological Specimen Preparation for Transmission Electron Microscopy, Princeton University Press, Princeton, NJ, 1998.

3. J. H. Luft, J. Biophys. Biochem. 9(1961)409.

4. E. A. Ellis, Proc. Microsc. Microanal. 8, suppl. 2 (2002)884CD.

5. A. R. Spurr, J. Ultrastruct. Res. 26(1969)31. 


\section{Eliminate the}

\section{Element of Surprise}

Advanced

EDS Technology

Accurately Identifies What's Really There

- Easily identify overlapping peaks-

Graphics assist in visualizing results

- Ensure accurate identification and quantification under variable pressure conditions-Exclusive software package compensates for SEM beam spread

- Identify unknown small phases and trace elementsNew data mining tool rapidly searches spectral map data sets to find elements at pixel level resolution

- Exclusive Seamless Integration Package for EDS, EBSD, and WDS-Provides comprehensive solution with easy expandability

\section{Results with Confidence}

As the world's leader in Electron Beam Microanalysis, EDAX continues to raise the performance standard with innovative features that are easy to adapt and provide results that you can trust to be complete and accurate.

For more information on our newest developments which ensure the accurate identification of even the most complex samples visit our web site at www.EDAX.com/update or call 1.201.529.4880

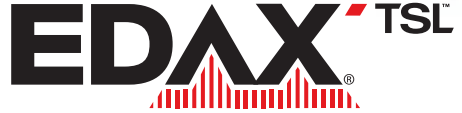

advanced microanalysis solutions

AMETEK 